\title{
Evaluating the Calibration and Power of Three Gene-Based Association Tests of Rare Variants for the X Chromosome
}

\author{
Clement Ma, Michael Boehnke, Seunggeun Lee, ${ }^{*}$ and the GoT2D Investigators \\ Department of Biostatistics and Center for Statistical Genetics, University of Michigan, Ann Arbor, Michigan, United States of America
}

Received 13 April 2015; Revised 11 August 2015; accepted revised manuscript 2 September 2015

Published online 10 October 2015 in Wiley Online Library (wileyonlinelibrary.com). DOI 10.1002/gepi.21935

\begin{abstract}
Although genome-wide association studies (GWAS) have identified thousands of trait-associated genetic variants, there are relatively few findings on the $\mathrm{X}$ chromosome. For analysis of low-frequency variants (minor allele frequency $<5 \%$ ), investigators can use region- or gene-based tests where multiple variants are analyzed jointly to increase power. To date, there are no gene-based tests designed for association testing of low-frequency variants on the X chromosome. Here we propose three gene-based tests for the X chromosome: burden, sequence kernel association test (SKAT), and optimal unified SKAT (SKAT-O). Using simulated case-control and quantitative trait (QT) data, we evaluate the calibration and power of these tests as a function of (1) male:female sample size ratio; and (2) coding of haploid male genotypes for variants under X-inactivation. For case-control studies, all three tests are reasonably well-calibrated for all scenarios we evaluated. As expected, power for gene-based tests depends on the underlying genetic architecture of the genomic region analyzed. Studies with more (haploid) males are generally less powerful due to decreased number of chromosomes. Power generally is slightly greater when the coding scheme for male genotypes matches the true underlying model, but the power loss for misspecifying the (generally unknown) model is small. For QT studies, type I error and power results largely mirror those for binary traits. We demonstrate the use of these three gene-based tests for X-chromosome association analysis in simulated data and sequencing data from the Genetics of Type 2 Diabetes (GoT2D) study.

Genet Epidemiol 39:499-508, 2015. @ 2015 Wiley Periodicals, Inc.
\end{abstract}

KEY WORDS: rare variants; low-frequency variants; gene-based association tests; genome-wide association study

\section{Introduction}

Genome-wide association studies (GWAS) have identified thousands of genetic variants associated with hundreds of diseases and traits [Hindorff et al., 2012]. However, the proportion of associated variants on the $\mathrm{X}$ chromosome, relative to its chromosomal length, lags far behind those on the autosomes [Wise et al., 2013]. Analysis of X-chromosome association requires proper treatment of diploid female and haploid male participants. Although we can code female genotypes under an additive model as the number of minor alleles for a specific variant: $g=\{0,1,2\}$, just as we do for autosomal variants, for male genotypes, there are two obvious coding schemes. For a variant under X-inactivation [Lyon, 1961], where one copy of the female $\mathrm{X}$ chromosome is inactivated, one copy of the male allele is equivalent to two copies of the female allele, and hence we code haploid male genotypes as $g=\{0,2\}$. For a variant at a locus that does not undergo $\mathrm{X}$-inactivation, we code male genotypes as $g=\{0,1\}$. For analysis of a mixed sample of males and females, specialized analytical tools are needed for initial data processing (e.g., es-

Supporting Information is available in the online issue at wileyonlinelibrary.com.

* Correspondence to: Seunggeun Lee, Department of Biostatistics and Center for Statistical Genetics, University of Michigan, M4148 SPH II, 1415 Washington Heights, Ann Arbor, MI 48109-2029. E-mail: leeshawn@umich.edu timating allele frequencies and testing Hardy-Weinberg equilibrium) [Purcell et al., 2007], genotype imputation [Howie et al., 2012; Marchini et al., 2007], and association analysis [Clayton, 2008; Zheng et al., 2007]. Hence, in many GWAS, the analysis of the $\mathrm{X}$ chromosome has been omitted due to the additional analysis steps required and/or lack of available software tools [Wise et al., 2013]. With use of specialized analytical tools, additional trait-associated variants on the $\mathrm{X}$ chromosome are likely to be identified.

Existing X-chromosome analysis methods focus on singlemarker association analysis. Zheng et al. [2007] proposed tests comparing differences in allele counts between cases and controls for males and females jointly, and assume no $\mathrm{X}$-inactivation (coding male genotypes as $g=\{0,1\}$ ). Clayton [2008] proposed score tests for the additive and dominant genetic models assuming X-inactivation (coding male genotypes as $g=\{0,2\}$ ). His test assumes equal allele frequencies in males and females; if this assumption is violated, he recommended stratifying by sex and combining score statistics across strata. Loley et al. [2011] evaluated the calibration and power of these tests and showed that no single test is uniformly most powerful over all genetic models and simulation parameters. Loley demonstrated that Clayton's nonsex-stratified tests can be anti-conservative when allele frequencies differ between the sexes. Hickey and Bahlo 
[2011] conducted a similar evaluation, and showed that tests that made use of both male and female data were uniformly more powerful than tests that only use female data.

Many recent genetic studies use genome or exome sequencing [Steinthorsdottir et al., 2014] or specialized genotyping arrays [Huyghe et al., 2013] to better assay low-frequency genetic variants (minor allele frequency $[\mathrm{MAF}]<5 \%$ ). Singlemarker tests have low power to test for association with lowfrequency variants unless the sample and/or effect size is very large [Asimit and Zeggini, 2010]. In contrast, region- or genebased tests in which multiple markers are analyzed jointly can be more powerful for analyzing low-frequency variants [Lee et al., 2014]. The calibration and power of gene-based tests have not been evaluated in the context of analyzing low-frequency variants on the $\mathrm{X}$ chromosome. In this paper, we describe, apply, and evaluate three gene-based tests for the X chromosome: burden, sequence kernel association test (SKAT), and optimal unified SKAT (SKAT-O) [Lee et al., 2012]. Specifically, using simulated binary and quantitative trait (QT) datasets, we evaluate the calibration and power of these tests with (1) different male:female ratios in cases and controls, and (2) different coding of male genotypes.

We find that for case-control studies, all tests are wellcalibrated or very slightly anti-conservative for different male:female ratios in cases and controls, and different coding of male genotypes. As expected, power depends on the underlying genetic architecture of the genomic region analyzed. Studies with more males than females are typically less powerful. In most scenarios, power is slightly greater when we analyze data assuming the true model to code male genotypes. For QT studies, burden and SKAT are well-calibrated, while SKAT-O can be slightly anti-conservative, and power results are similar to those for binary traits. We conclude that these gene-based tests can be directly applied to the association analysis of low-frequency variants for both binary and QTs. We implemented these tests in the SKAT R package [Lee, 2014].

\section{Methods}

\section{Notation}

Consider $n$ individuals sequenced at $m$ variants in a genomic region of interest. For individual $i$, let $\mathbf{X}_{\mathbf{i}}=$ $\left(x_{i 1}, \ldots, x_{i s}\right)^{\prime}$ be the vector of $s$ covariates (including a covariate for sex $)$ and $\mathbf{G}_{\mathbf{i}}=\left(g_{i 1}, \ldots, g_{i m}\right)^{\prime}$ be the vector of genotypes. For (diploid) females, let $g_{i j}=\{0,1,2\}$ be the number of minor alleles for variant $j$. For (haploid) males, we consider two coding schemes: (1) $g_{i j}=\{0,2\}$ when assuming Xinactivation in the females and (2) $g_{i j}=\{0,1\}$ when assuming no X-inactivation. For binary traits, $y_{i}=1$ or $y_{i}=0$ denotes a case or control, respectively; for QTs, $y_{i}$ denotes the QT value. In a combined sample of $n_{m}$ males and $n_{f}$ females (all unrelated), the maximum-likelihood estimate of the MAF $p$ of a biallelic variant with alleles $A$ and $a$ is

$$
\hat{p}=\frac{2 n_{a a, f}+n_{A a, f}+n_{a, m}}{2 n_{f}+n_{m}},
$$

where $n_{a a, f}$ and $n_{A a, f}$ are the number of females with genotypes $a a$ and $A a$, and $n_{a, m}$ is the number of males with the $a$ allele.

\section{Gene-Based Tests}

For binary traits, we consider the logistic regression model:

$$
\operatorname{logit}\left(\operatorname{Pr}\left(y_{i}=1\right)\right)=\operatorname{logit}\left(\pi_{i}\right)=\gamma_{0}+\mathbf{X}_{\mathbf{i}}^{\prime} \gamma_{1}+\mathbf{G}_{\mathbf{i}}^{\prime} \beta,
$$

where $\gamma_{0}$ is the intercept, $\gamma_{1}$ is the $s \times 1$ vector of regression coefficients for the covariates, and $\beta=\left(\beta_{1}, \ldots, \beta_{m}\right)^{\prime}$ is the $m \times 1$ vector of regression coefficients for the genetic variants. For QTs, the linear regression model is

$$
y_{i}=\gamma_{0}+\mathbf{X}_{\mathbf{i}}^{\prime} \gamma_{1}+\mathbf{G}_{\mathbf{i}}^{\prime} \beta+\varepsilon_{i},
$$

where $\varepsilon_{i}$ is the normally distributed error term with mean zero and variance $\sigma^{2}$.

Because there is limited power to test the null hypothesis that the vector $\boldsymbol{\beta}=0$ for large $m$, the burden test combines the genetic effects over the genomic region by assuming $\beta_{j}=$ $w_{j} \beta_{c}$, given weights $w_{j}$. Thus, Equations (1) and (2) become

$$
\begin{gathered}
\operatorname{logit}\left(\pi_{i}\right)=\gamma_{0}+\mathbf{X}_{{ }_{\mathbf{i}}}^{\prime} \gamma_{1}+\beta_{c}\left(\sum_{j=1}^{m} w_{j} g_{i j}\right) \\
y_{i}=\gamma_{0}+\mathbf{X}_{\mathbf{i}}^{\prime} \gamma_{1}+\beta_{c}\left(\sum_{j=1}^{m} w_{j} g_{i j}\right)+\varepsilon_{i}
\end{gathered}
$$

We recommend using MAF-based weights to upweight low-frequency variants: $w_{j}=\operatorname{Beta}\left(\hat{p}_{j}, \alpha=1, \beta=\right.$ $25)$, where weights have beta density function with prespecified parameters $\alpha$ and $\beta$, and $\hat{p}_{j}$ is the variant MAF [Wu et al., 2011]. In simulations and real data analysis, we use $\alpha=1$ and $\beta=25$ as suggested by Wu et al. [2011]. To test the gene-based null hypothesis $H_{o}: \beta_{c}=0$, the burden score statistic is

$$
Q_{B}=\left(\sum_{j=1}^{m} w_{j} S_{j}\right)^{2},
$$

where $S_{j}=\sum_{i=1}^{n}\left(y_{i}-\hat{\mu}_{i}\right) g_{i j}$ is the score statistic for testing $H_{o}: \beta_{j}=0$ with only variant $j$ in the regression model, and $\hat{\mu}_{i}$ is the estimated mean of $y_{i}$ under $H_{o}$. The burden score statistic is evaluated relative to a scaled $\chi_{1}^{2}$ distribution [Wu et al., 2011].

SKAT assumes the $\beta_{j}$ 's follow an arbitrary distribution with mean zero and variance $w_{j}^{2} \tau$. Testing the null hypothesis $H_{o}$ : $\boldsymbol{\beta}=0$ is equivalent to testing $H_{0}: \tau=0$. The SKAT score statistic is

$$
Q_{S}=\sum_{j=1}^{m} w_{j}^{2} S_{j}^{2}
$$

and follows a mixture of chi-square distributions [Lee et al., 2012].

Wu et al. [2011] showed that the power of the burden test and SKAT depends on the underlying genetic architecture of 
the analyzed genomic region. For example, the burden test is more powerful when most variants in the region are causal and have the same direction of effect; in contrast, SKAT is more powerful when fewer variants are causal and/or have opposite directions of effect. The combined test of burden test and SKAT, SKAT-O [Lee et al., 2012] combines the strength of both tests and is powerful in both scenarios. The SKAT-O statistic is a weighted average of $Q_{B}$ and $Q_{S}$ :

$$
Q_{\rho}=\rho Q_{B}+(1-\rho) Q_{S}, \quad 0 \leq \rho \leq 1
$$

with weight parameter $\rho$. In practice, $\rho$ is unknown. To estimate the optimal $\rho$, we perform a grid search on $0=\rho_{1}<$ $\rho_{2}<\cdots<\rho_{b}=1$ and select $\rho$ such that the $Q_{\rho}$ is maximized (or the corresponding $P$-value is minimized). We choose to perform the search on $\rho=\left\{0,0.1^{2}, 0.2^{2}, 0.3^{2}, 0.4^{2}, 0.5^{2}, 0.5\right.$, $1\}$ as suggested by Lee et al. [2012]. Significance is evaluated analytically by numerical integration [Lee et al., 2012].

For analysis of case-control studies, we examine the effect of applying the small-sample adjustment described in Lee et al. [2012].

\section{Numerical Simulations}

To generate simulated genomic regions, we used 10,000 haplotypes simulated using the COSI coalescent simulator [Schaffner et al., 2005], as provided in the SKAT R package. For each simulated male individual, we randomly selected a $3 \mathrm{~Kb}$ region from a single random haplotype. For each simulated female individual, we selected a $3 \mathrm{~Kb}$ region from two random haplotypes and paired them together. For a simulated sample of 1,000 cases and 1,000 controls under the null hypothesis, the $3 \mathrm{~Kb}$ region has average number of variants $=36.8(\mathrm{SD}=6.0)$, with a median total minor allele count $[\mathrm{MAC}]=2,812$ (interquartile range $[\mathrm{IQR}]=1,766-4,270$ ). When considering only variants with $\mathrm{MAF}<0.01$, the average number of variants $=28.4(\mathrm{SD}=5.2)$, with median total $\mathrm{MAC}=99(\mathrm{IQR}=79-125)$.

\section{Type I Error Simulations}

For binary traits, we simulated case-control datasets with $N_{\text {cases }}=1,000$ and $N_{\text {ctrls }}=1,000$ under the logistic regression model:

$$
\operatorname{logit}\left(\pi_{i}\right)=\gamma_{0}+\gamma_{1} X_{1 i}+\gamma_{2} X_{2 i}+\gamma_{3} X_{3 i}+\beta_{1} g_{i 1}+\cdots+\beta_{t} g_{i t}
$$

with one continuous covariate $X_{1 i}$ normally distributed with mean zero and variance one, one binary covariate $X_{2 i}$ distributed Bernoulli with success probability $f=0.5$, sex covariate $X_{3 i}$, and selected causal variants $g_{i 1}, \ldots, g_{i t}$; under the null model, we set genetic effects $\beta_{1}=\cdots=\beta_{t}=0$. The sex covariate accounts for differences in genotype frequency between males and females, so that we can avoid inflated type I error rates as for Clayton's score test [2008] when allele frequencies differ between sexes [Loley et al., 2011]. We set the intercept $\gamma_{0}$ so that the disease prevalence is $10 \%$, the covariate regression coefficients $\gamma_{1}=\gamma_{2}=0.5$, and the effect for
Table 1. Sample sizes for simulated case-control datasets

\begin{tabular}{lrc}
\hline Simulation & No. of cases males:females & No. of controls males:females \\
\hline A & $500: 500$ & $500: 500$ \\
B & $900: 100$ & $500: 500$ \\
C & $100: 900$ & $500: 500$ \\
\hline
\end{tabular}

Table 2. Sample sizes for simulated quantitative trait datasets

\begin{tabular}{lc}
\hline Simulation & No. of individuals males:females \\
\hline D & $1,000: 1,000$ \\
E & $200: 1,800$ \\
F & $1,800: 200$ \\
\hline
\end{tabular}

sex $\gamma_{3}=0$. We explored a broad range of male:female ratios in cases and controls by sampling the exact number of males and females (Table 1) from the simulated cases and controls. Note that there is an implicit sex-phenotype effect when we sample unbalanced numbers of males and females in cases and controls in simulation scenarios B and C (Table 1).

For QTs, we took a similar approach to simulate datasets of $N=2,000$ individuals under the null linear regression model:

$$
y_{i}=\gamma_{0}+\gamma_{1} X_{1 i}+\gamma_{2} X_{2 i}+\gamma_{3} X_{3 i}+\beta_{1} g_{i 1}+\cdots+\beta_{t} g_{i t}+\varepsilon_{i}
$$

with $X_{1 i}, X_{2 i}, X_{3 i}$, and $g_{i 1}, \ldots, g_{i t}$ as for Equation (8). We set covariate effect sizes $\gamma_{1}=\gamma_{2}=0.5$, or equivalently, the proportion of trait variance explained $\sigma_{X 1}^{2}=\left(\gamma_{1}\right)^{2}$ and $\sigma_{X 2}^{2}=\left(\gamma_{2}\right)^{2} f(1-f)$; we set the effect of sex $\gamma_{3}=0$ or 0.5 . The normally distributed residual error had mean zero and variance $=1-\sigma_{X 1}^{2}-\sigma_{X 2}^{2}$. We sampled the desired number of males and females (Table 2) from the simulated individuals.

We analyzed each simulated dataset using the six combinations of three gene-based tests (Eqs. (5)-(7)) and two coding schemes for male genotypes. To increase computational efficiency, we simulated 100,000 independent datasets per simulation scenario, and resampled the phenotype 1,000 times per independent dataset, resulting in a total of 100 million simulation replicates. We evaluated the robustness of the resampling approach by comparing results with 1 million independent simulated datasets without resampling for a subset of the simulation scenarios. We estimated type I error as the proportion of simulation replicates with a $P$-value $<\alpha=2.5 \times 10^{-6}$, corresponding to Bonferroni correction for association testing of the approximately 20,000 genes in the human genome.

\section{Power Simulations}

Within the $3 \mathrm{~Kb}$ region, we selected $10 \%$ or $50 \%$ of variants with $\mathrm{MAF}<0.03$ as causal. Using the same settings as for the type I error simulations, we simulated case-control datasets using the logistic regression model (Eq. (8)). We simulated QT datasets using the linear regression model (Eq. (9)), assuming the normally distributed residual error $\varepsilon_{i}$ had mean zero and variance $=1-\sigma_{X 1}^{2}-\sigma_{X 2}^{2}-\sum_{j=1}^{t} \sigma_{j}^{2}$, where 
Table 3. Type I error rates for burden, SKAT, and SKAT-O tests for simulated binary and quantitative trait studies

Binary traits

\begin{tabular}{|c|c|c|c|c|c|c|}
\hline \multirow[b]{2}{*}{ Simulation } & \multirow[b]{2}{*}{ No. of cases males:females } & \multirow[b]{2}{*}{ No. of controls males:females } & \multirow[b]{2}{*}{ Coding for male genotypes } & \multicolumn{3}{|c|}{ Type I error rate $\left(\times 10^{-6}\right)$} \\
\hline & & & & Burden & SKAT & SKAT-O \\
\hline \multirow[t]{2}{*}{ A } & \multirow[t]{2}{*}{$500: 500$} & \multirow[t]{2}{*}{$500: 500$} & No X-inactivation $g=\{0,1\}$ & 2.4 & 2.6 & 2.4 \\
\hline & & & $\mathrm{X}$-inactivation $g=\{0,2\}$ & 1.8 & 1.9 & 1.6 \\
\hline \multirow[t]{2}{*}{ B } & \multirow[t]{2}{*}{$900: 100$} & \multirow[t]{2}{*}{$500: 500$} & No X-inactivation $g=\{0,1\}$ & 3.1 & 3.9 & 2.9 \\
\hline & & & X-inactivation $g=\{0,2\}$ & 1.8 & 2.9 & 2.1 \\
\hline \multirow[t]{2}{*}{$\mathrm{C}$} & \multirow[t]{2}{*}{$100: 900$} & \multirow[t]{2}{*}{$500: 500$} & No X-inactivation $g=\{0,1\}$ & 3.1 & 3.4 & 3.0 \\
\hline & & & $X$-inactivation $g=\{0,2\}$ & 2.8 & 5.2 & 3.7 \\
\hline
\end{tabular}

Quantitative traits

\begin{tabular}{|c|c|c|c|c|c|}
\hline \multirow[b]{2}{*}{ Simulation } & \multirow[b]{2}{*}{ No. of individuals males:females } & \multirow[b]{2}{*}{ Coding for male genotypes } & \multicolumn{3}{|c|}{ Type I error rate $\left(\times 10^{-6}\right)$} \\
\hline & & & Burden & SKAT & SKAT-O \\
\hline \multirow[t]{2}{*}{$\mathrm{D}$} & $1,000: 1,000$ & No X-inactivation $g=\{0,1\}$ & 2.5 & 2.1 & 2.8 \\
\hline & & X-inactivation $g=\{0,2\}$ & 2.5 & 2.4 & 2.8 \\
\hline \multirow[t]{2}{*}{$\mathrm{E}$} & $1,800: 200$ & No X-inactivation $g=\{0,1\}$ & 2.7 & 2.5 & 3.3 \\
\hline & & $\mathrm{X}$-inactivation $g=\{0,2\}$ & 2.5 & 2.4 & 3.1 \\
\hline \multirow[t]{2}{*}{$\mathrm{F}$} & $200: 1,800$ & No X-inactivation $g=\{0,1\}$ & 2.6 & 2.8 & 3.4 \\
\hline & & $\mathrm{X}$-inactivation $g=\{0,2\}$ & 2.7 & 2.8 & 3.7 \\
\hline
\end{tabular}

Type I error estimates are based on $10^{8}$ simulation replicates so that the nominal significance threshold of $\alpha=2.5 \times 10^{-6}$ corresponds to 250 rejections. Empirical type I error rates between $2.2 \times 10^{-6}$ and $2.8 \times 10^{-6}$ have $95 \%$ confidence intervals that include the nominal value.

$\sigma_{j}^{2}=\left(\beta_{j}\right)^{2} 2 p_{j}\left(1-p_{j}\right)$ is the proportion of trait variance explained by variant $j$.

We simulated datasets under the alternative hypothesis assuming X-inactivation and non-X-inactivation coding for male genotypes. We assumed genetic effect sizes proportional to the variant $\operatorname{MAF}\left|\beta_{j}\right|=c\left|\log _{10} p_{j}\right| / 2$, and adjusted the tuning parameter $c$ so that power estimates were not too close to 1 or 0 . For binary traits, when $10 \%$ of variants were causal, we set $c=\log (15)$, to give an odds ratio of 15 when $\mathrm{MAF}=0.01$; when $50 \%$ variants were causal, we set $c=\log (3)$ or $\log (5)$. For QTs, when $10 \%$ of variants were causal, we set $c=\log (7)$, which gave a linear regression coefficient of approximately 1.95 when $\mathrm{MAF}=0.01$; when $50 \%$ of variants were causal, we set $c=\log (1.8)$. We assumed that either all causal variants were deleterious, or that $50 \%$ were deleterious and $50 \%$ were protective. We simulated 1,000 independent replicates per simulation scenario, and evaluated power as the proportion of replicates with $P$-value $<2.5 \times 10^{-6}$.

\section{Genetics of Type 2 Diabetes (GoT2D) Study}

To assess these methods in the context of real data, we analyzed integrated sequencing and genotyping data from the GoT2D study, which aims to investigate the impact of lowfrequency genetic variation on type 2 diabetes (T2D) risk. The GoT2D study sample is composed of 1,326 T2D cases and 1,331 normal glucose tolerant controls from the United Kingdom ( 322 cases / 322 controls), Finland (486/517), the Botnia region of Finland (199/159), Germany (104/101), and Sweden (222/227). There are 716 males and 610 females in cases and 592 males and 739 females in controls. For each sample, we performed low-pass $(\sim 5 \times)$ whole genome sequencing, augmented by deep $(\sim 100 \times)$ whole-exome sequencing, and genotyping of $2.5 \mathrm{M}$ single nucleotide polymorphisms (SNPs) using the Illumina HumanOmni2.5 array. To mimic the type I error and power simulations, we analyzed X-chromosome data using the six combinations of three gene-based tests and two coding schemes for male genotypes. For each test, we adjusted for the effects of sex, the first two genotype-based principal components (PCs) to control for population stratification [Price et al., 2006], and indicator functions for observed temporal stratification based on sequencing date and center. To filter out likely neutral variants from the analysis, we restricted the gene-based analysis to protein-truncating and missense variants. We used the seqminer [Zhan and Liu, 2013] and SKAT [Lee, 2014] R packages to extract the genotypes and perform the gene-based analysis, respectively.

\section{Results}

\section{Type I Error Rates}

For binary traits, the burden, SKAT, and SKAT-O tests are well-calibrated or slightly anti-conservative (at $\alpha=2.5 \times$ $10^{-6}$ ) for all scenarios considered (Table 3 ). For each genebased test, we examined type I error rates for two male genotype coding schemes: (1) $g_{i j}=\{0,1\}$ and (2) $g_{i j}=\{0,2\}$; and datasets with three male:female ratios in cases: (Simulation A) 500:500, (B) 900:100, and (C) 100:900. For datasets with male:female ratio $=500: 500$ in cases (Simulation A), non-Xinactivation coding is less conservative than $\mathrm{X}$-inactivation coding for all three tests: burden (non-X-inactivation type I error rate $=2.4 \times 10^{-6}$ vs. X-inactivation $\left.=1.8 \times 10^{-6}\right)$, SKAT $\left(2.6 \times 10^{-6}\right.$ vs. $\left.1.9 \times 10^{-6}\right)$, and SKAT-O $\left(2.4 \times 10^{-6}\right.$ vs. $\left.1.6 \times 10^{-6}\right)$. These patterns also hold true for datasets with male:female ratio $=900: 100$ in cases (Simulation B). In comparison, for datasets with male:female ratio $=100: 900$ in cases (Simulation $\mathrm{C}$ ), non-X-inactivation coding is less 

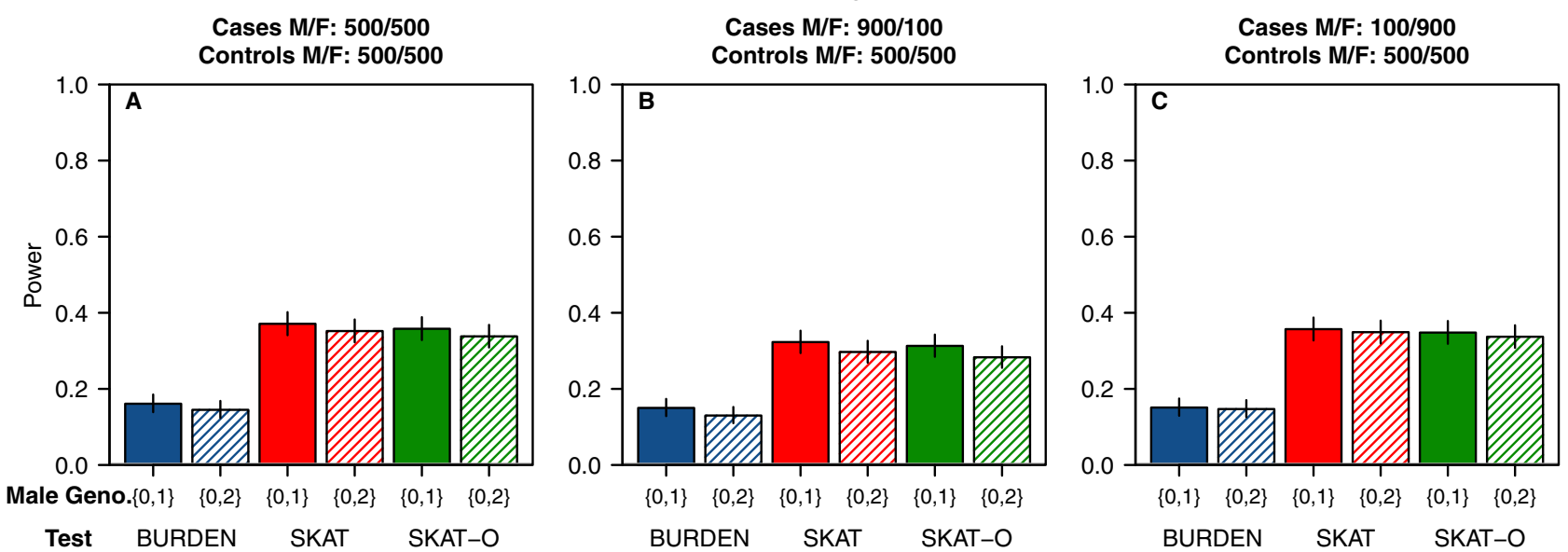

$50 \%$ Causal Variants in Region (100\% Deleterious)
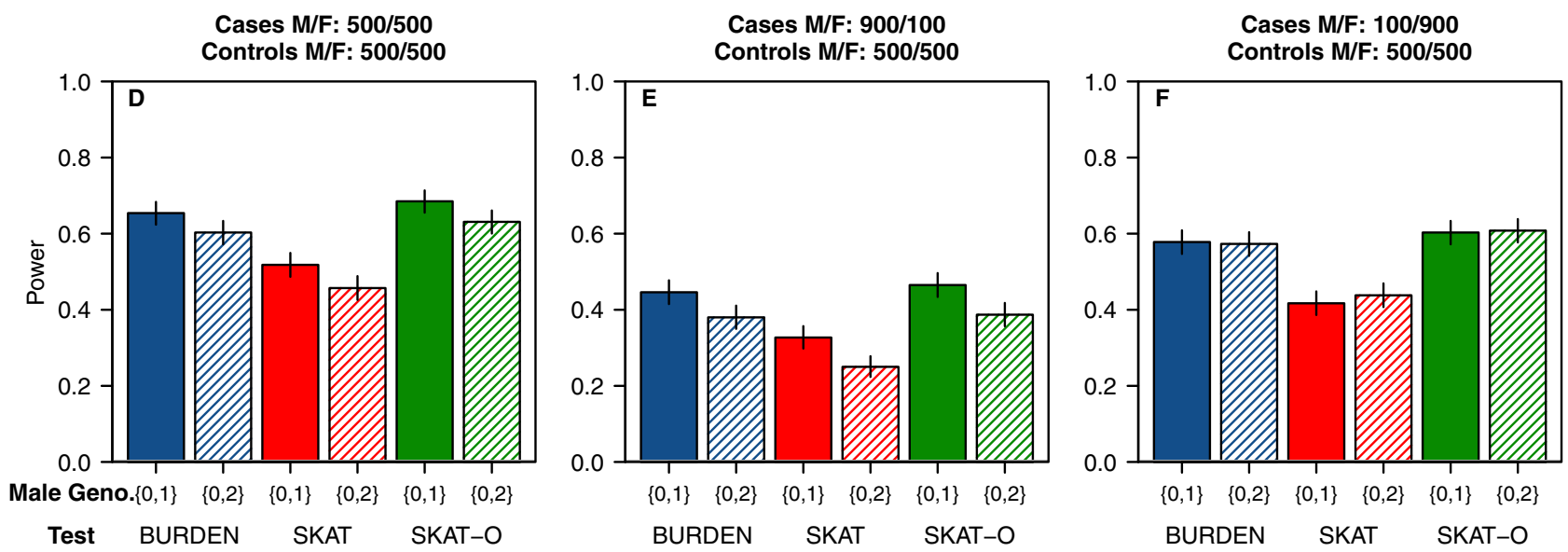

Figure 1. Power for gene-based tests in case-control studies assuming all causal variants are deleterious. Causal variants are simulated with non-X-inactivation coding of male genotypes $g_{i j}=\{0,1\}$. Within each simulated $3 \mathrm{~Kb}$ genomic region, (A-C) $10 \%$ or (D-F) $50 \%$ of variants with MAF $<0.03$ are selected as causal. The effect size for causal variants is given by $\left|\beta_{j}\right|=c\left|\log _{10} p_{j}\right| / 2$, and is proportional to MAF $\left(p_{j}\right)$ and scaled by tuning parameter $(A-C) c=\log (15)$ and $(D-F) c=\log (3)$; all causal variants are simulated as deleterious. Simulated cases have $(A, D) 500: 500,(B, E)$ 900:100, and (C, F) 100:900 males and females, respectively. All simulated controls have 500 males and 500 females. Power estimates (at $\alpha=2.5 \times$ $\left.10^{-6}\right)$ are based on 1,000 simulation replicates; vertical bars denote the exact binomial $95 \%$ confidence intervals.

conservative for burden, but more conservative for SKAT and SKAT-O. These results are generalizable to other male:female sample sizes and depend only on the male:female ratio in cases and controls (supplementary Fig. S1).

We examined the impact of the implicit nonzero sexphenotype effect on test calibration by sampling unbalanced numbers of males and females in cases and controls (Simulations B and C; Table 1). Tests are similarly calibrated with or without the sex-phenotype effect (Table 3). We also examined the effect of applying the small-sample adjustment [Lee et al., 2012] to the three gene-based tests; type I error rates are generally slightly anti-conservative after applying the smallsample adjustment (supplementary Fig. S1), but the patterns of type I error rates between male genotype coding schemes and male:female ratios are identical to those without smallsample adjustment. Finally, we demonstrated the accuracy of our computationally efficient resampling approach by com- paring type I error rates with resampling to those without resampling $\left(10^{6}\right.$ independent replicates; $\left.\alpha=5 \times 10^{-4}\right)$; type I error rates are comparable with (supplementary Fig. S2A-C) and without resampling (supplementary Fig. S2D-F).

For QTs, the burden and SKAT tests are well-calibrated and SKAT-O can be very slightly anti-conservative across the three simulated datasets with male:female ratios of 1,000:1,000; 1,800:200; and 200:1,800 (Table 3). Type I error rates are nearly identical between the two male coding schemes. Tests are similarly well-calibrated with or without the inclusion of a nonzero sex-phenotype effect (supplementary Table S1).

\section{Power}

We examined power for four combinations of proportion of causal variants in a region ( $10 \%$ or $50 \%)$ and causal variant direction of effect (all deleterious, or 50\% deleterious and 

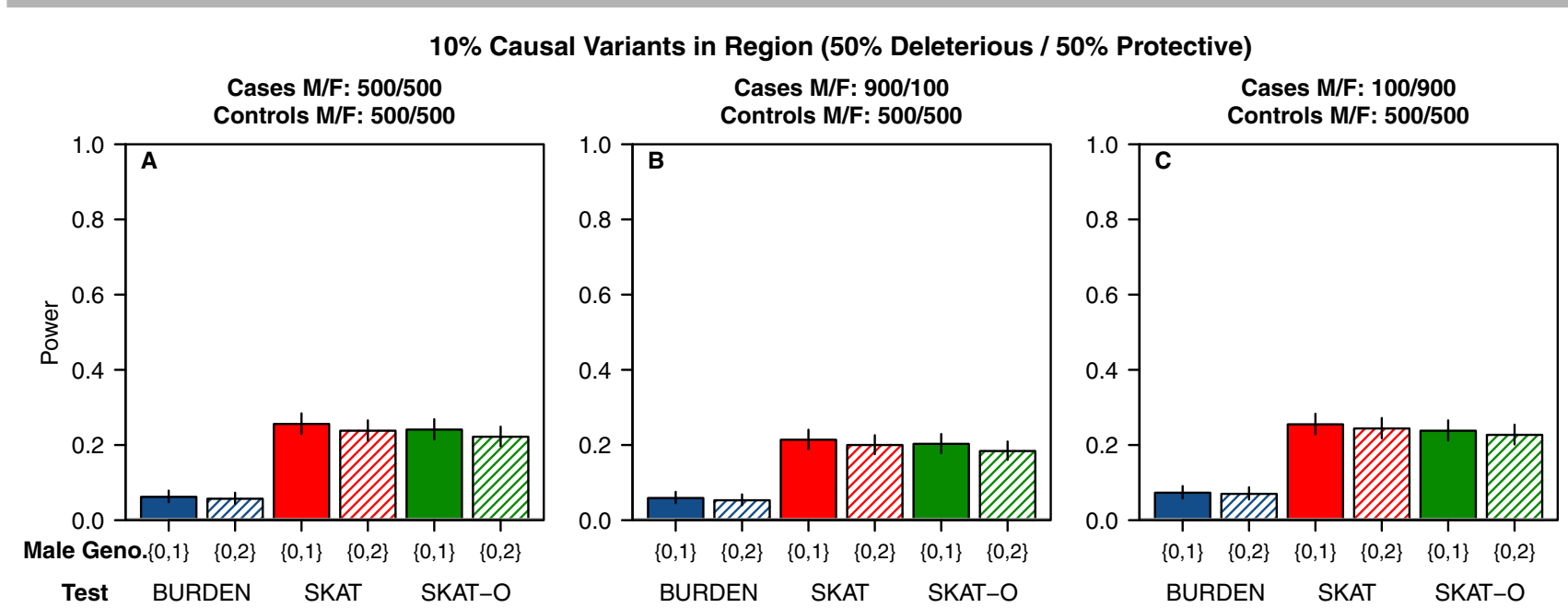

$50 \%$ Causal Variants in Region (50\% Deleterious / 50\% Protective)
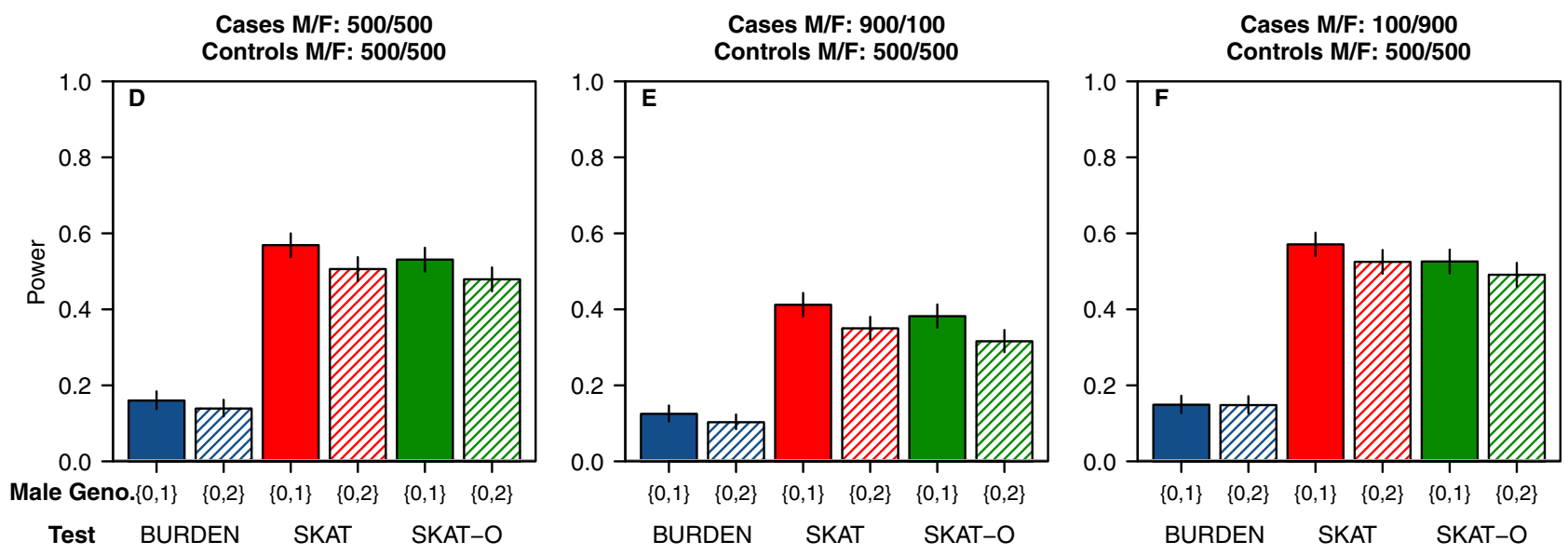

Figure 2. Power for gene-based tests in case-control studies assuming causal variants are $50 \%$ deleterious and $50 \%$ protective. Causal variants are simulated with non-X-inactivation coding of male genotypes $g_{i j}=\{0,1\}$. Within each simulated $3 \mathrm{~Kb}$ genomic region, (A-C) $10 \%$ or (D-F) $50 \%$ of variants with MAF $<0.03$ are selected as causal. The effect size for causal variants is given by $\left|\beta_{j}\right|=c\left|\log _{10} p_{j}\right| / 2$, and is proportional to MAF $\left(p_{j}\right)$ and scaled by tuning parameter $(A-C) c=\log (15)$ and $(D-F) c=\log (5)$; causal variants are simulated as $50 \%$ deleterious and $50 \%$ protective. Simulated cases have (A, D) 500:500, (B, E) 900:100, and (C, F) 100:900 males and females, respectively. All simulated controls have 500 males and 500 females. Power estimates (at $\alpha=2.5 \times 10^{-6}$ ) are based on 1,000 simulation replicates; vertical bars denote the exact binomial $95 \%$ confidence intervals.

$50 \%$ protective). For binary traits, power results (Figs. 1 and 2) reflect the previously described relative power of genebased tests for different underlying genetic architectures [Lee et al., 2012]. For example, the burden test is more powerful when $50 \%$ of low-frequency variants are causal and have the same direction of effect (Fig. 1D-F). SKAT is more powerful when $10 \%$ of low-frequency variants are causal with the same or opposite direction of effect (Fig. 1A-C), or when 50\% of causal variants have opposite direction of effect (Fig. 2). SKAT-O is generally robust and powerful across all scenarios tested. Despite the slightly anti-conservative type I error rates, the small-sample adjusted and nonadjusted power estimates are comparable (data not shown).

Next, we investigated the effect of simulating causal variants with (male genotype coding $\left.g_{i j}=\{0,2\}\right)$ and without $\left(g_{i j}\right.$ $=\{0,1\}) X$-inactivation. The two coding schemes for male genotypes have only a small effect on power. When simulating variants assuming no $\mathrm{X}$-inactivation, non-X-inactivation coding $\left(g_{i j}=\{0,1\}\right)$ is slightly more powerful in all scenarios (Figs. 1 and 2). However, when simulating variants assuming $\mathrm{X}$-inactivation, $\mathrm{X}$-inactivation coding $\left(g_{i j}=\{0,2\}\right)$ is slightly more powerful in nearly all scenarios (supplementary Figs. S3 and S4). However, the power loss for misspecifying the unknown model is small. For example, in simulations assuming non-X-inactivation coding, the largest power loss for misspecifying the coding scheme is $7.7 \%$ (32.7\% vs. $25.0 \%)$ for SKAT (Fig. 1E), and the average absolute difference is $2.8 \%$. Power is generally lower for studies with more males than females, due to decreased effective sample size (e.g., number of chromosomes). For example, for the burden test with nonX-inactivation coding $\left(g_{i j}=\{0,1\}\right)$, studies with 900 males: 100 females in cases is less powerful than with 500 males: 


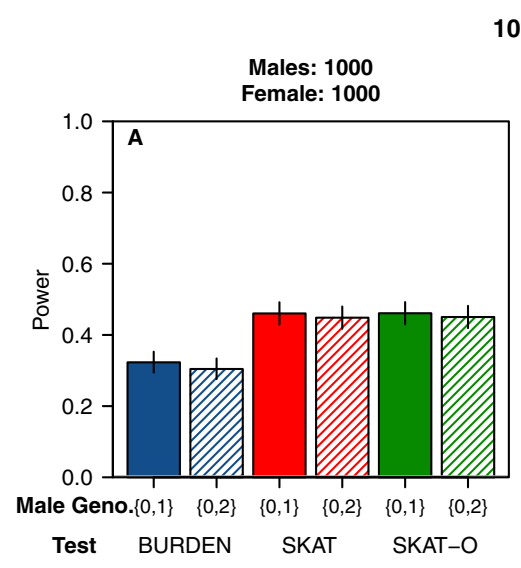

$10 \%$ Causal Variants in Region (100\% Deleterious)
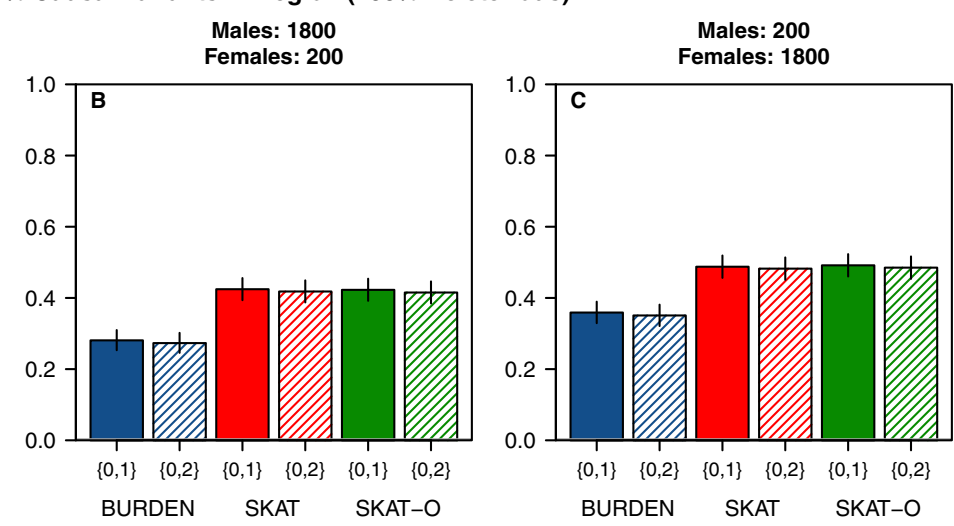

$50 \%$ Causal Variants in Region (100\% Deleterious)
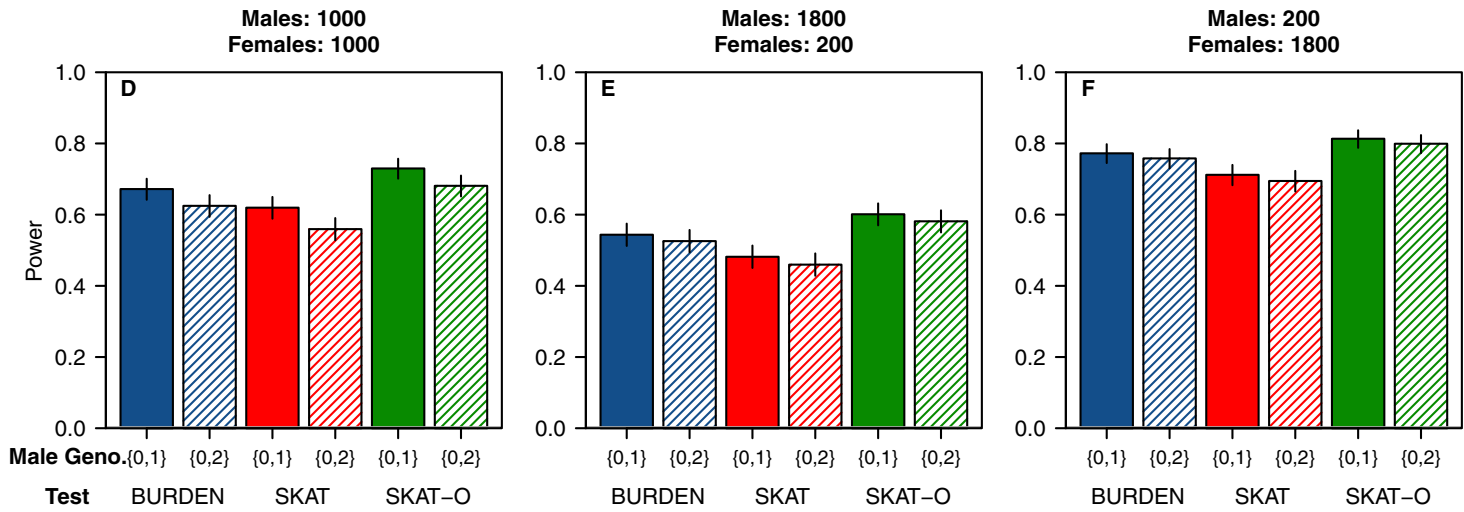

Figure 3. Power for gene-based tests in $\mathrm{OT}$ studies assuming all causal variants are deleterious. Causal variants are simulated with non-Xinactivation coding of male genotypes $g_{i j}=\{0,1\}$. Within each simulated $3 \mathrm{~Kb}$ genomic region, $(\mathrm{A}-\mathrm{C}) 10 \%$ or $(\mathrm{D}-\mathrm{F}) 50 \%$ of variants with $\mathrm{MAF}<0.03$ are selected as causal. The effect size for causal variants is given by $\left|\beta_{j}\right|=c\left|\log _{10} p_{j}\right| / 2$, and is proportional to $\operatorname{MAF}\left(p_{j}\right)$ and scaled by tuning parameter $(A-C) c=\log (7)$ and $(D-F) c=\log (1.8)$; all causal variants are simulated as deleterious. Simulated datasets have $(A, D) 1,000: 1,000,(B$, E) 1,800:200, and (C, F) 200:1,800 males and females, respectively. Power estimates (at $\alpha=2.5 \times 10^{-6}$ ) are based on 1,000 simulation replicates; vertical bars denote the exact binomial $95 \%$ confidence intervals.

500 females (44.6\% vs. $65.4 \%$; Fig. $1 \mathrm{D}$ and 1E). Finally, for QTs, power comparisons are very similar to those for binary traits (Figs. 3 and 4 ).

\section{Analysis of GoT2D X-Chromosome Data}

To examine the effect of different coding schemes for male genotypes, we performed gene-based association testing for $505 \mathrm{X}$-chromosome genes with T2D risk using low-pass sequencing data from $1,326 \mathrm{~T} 2 \mathrm{D}$ cases and 1,331 healthy controls from the GoT2D study. Within each gene region, we restricted the variants to those predicted to be protein truncating or missense mutations. Gene regions had an average number of variants $=6.1(\mathrm{SD}=5.5)$, and a median total MAC $=9(\mathrm{IQR}=4-20)$. We analyzed the data using six combinations of three gene-based tests and two male genotype coding schemes, adjusting for the effects of sex and the first two PCs to account for population stratification.

Scatterplots and quantile-quantile plots show that the association analysis $P$-values are concordant between $\mathrm{X}$ inactivation and non-X-inactivation coding for all three tests (Fig. 5A-F). The differences in $P$-value between coding schemes are generally small (Fig. 5G-I). For example, for the burden test, $68.5 \%$ (346/505 genes) of the analysis $P$-values have an absolute difference of $0.1 \log _{10}$ units or less; 99.4\% (502/505) of $P$-values are within $0.5 \log _{10}$ units.

\section{Discussion}

We examined the calibration and power of the burden, SKAT, and SKAT-O gene-based association tests for analyzing the X chromosome in simulated binary and QT data. For binary traits, all tests are well-calibrated or slightly anticonservative for all simulation scenarios. Power differences reflected the previously described strengths and weaknesses of each test for analyzing regions with differing underlying genetic architectures. Power is usually slightly increased when we code male genotypes with the coding scheme that matches the underlying genetic model (e.g., with or without $\mathrm{X}$-inactivation), but power loss is modest when we misspecify the (unknown) coding scheme. Studies with more male samples typically have lower power. For QTs, the burden and 

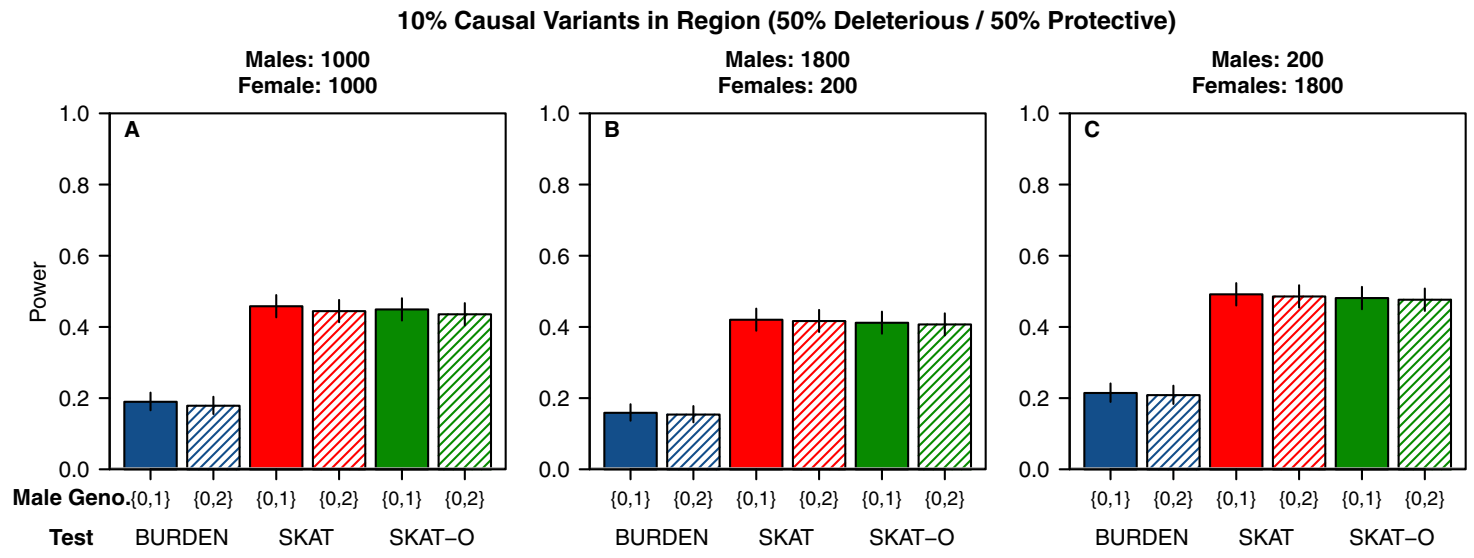

$50 \%$ Causal Variants in Region (50\% Deleterious / $50 \%$ Protective)
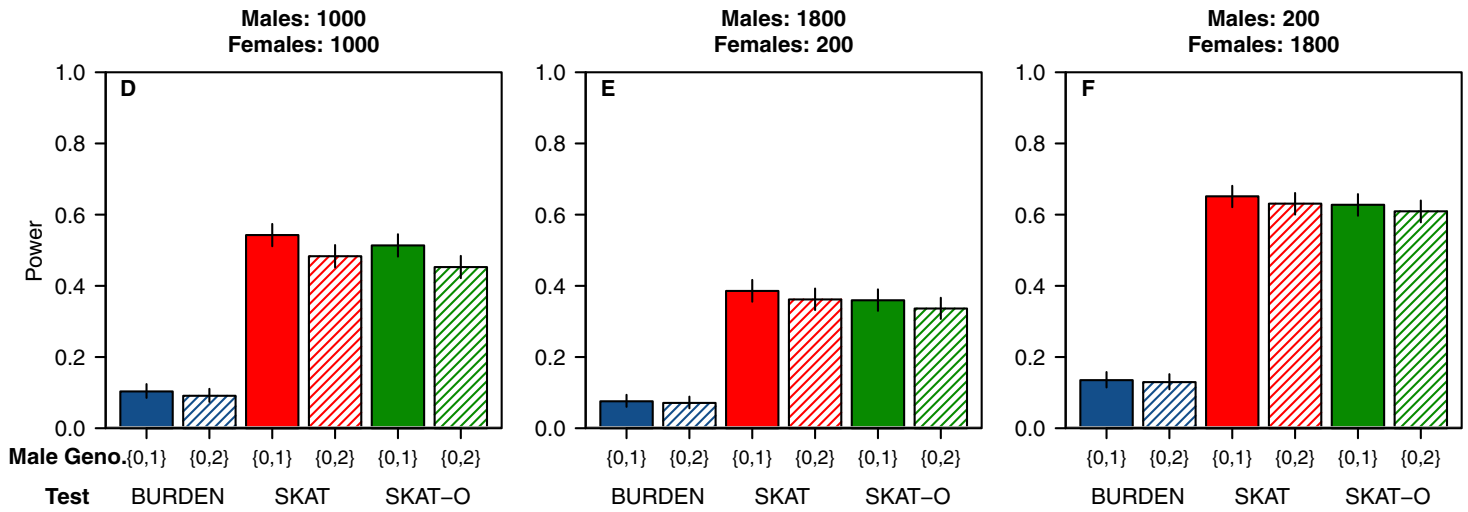

Figure 4. Power for gene-based tests in OT studies assuming causal variants are $50 \%$ deleterious and $50 \%$ protective. Causal variants are simulated with non-X-inactivation coding of male genotypes $g_{i j}=\{0,1\}$. Within each simulated $3 \mathrm{~Kb}$ genomic region, (A-C) $10 \%$ or $(\mathrm{D}-\mathrm{F}) 50 \%$ of variants with $\mathrm{MAF}<0.03$ are selected as causal. The effect size for causal variants is given by $\left|\beta_{j}\right|=c\left|\log _{10} p_{j}\right| / 2$, and is proportional to MAF $\left(p_{j}\right)$ and scaled by tuning parameter $(\mathrm{A}-\mathrm{C}) c=\log (7)$ and $(\mathrm{D}-\mathrm{F}) c=\log (1.8)$; causal variants are simulated as $50 \%$ deleterious and $50 \%$ protective. Simulated datasets have (A, D) 1,000:1,000, (B, E) 1,800:200, and (C, F) 200:1,800 males and females, respectively. Power estimates (at $\alpha=2.5 \times 10^{-6}$ ) are based on 1,000 simulation replicates; vertical bars denote the exact binomial $95 \%$ confidence intervals.

SKAT tests are well-calibrated, and SKAT-O is very slightly anti-conservative; power results mirror those for binary traits.

In practice, investigators will need to use external biological information to select the preferred male coding scheme. For example, we recommend using the non-X-inactivation coding scheme for known pseudoautosomal regions. In the absence of external information, we suggest using the $\mathrm{X}$ inactivation coding because a higher proportion of X-linked genes do undergo X-inactivation. It is known that only $15 \%$ of X-linked genes escape inactivation to some degree [Carrel and Willard, 2005]. We have demonstrated that misspecifying the coding scheme leads to only a small loss in power. In the GoT2D study, we also demonstrated that using either coding scheme produces similar results.

Although we only presented calibration and power results for a specific set of simulation settings, we performed a variety of simulations with other covariate settings, case:control ratios, and prevalence rates to demonstrate that our results are generalizable (data not shown). We also considered the effect on calibration of allele frequency differences between males and females. To introduce large differences in allele frequency between sexes, we sampled male genotypes from a set of simulated haplotypes mimicking European American ancestry, and female genotypes from haplotypes mimicking African American ancestry. The gene-based tests remain well-calibrated despite the allele frequency differences (supplementary Table S2).

We estimated nominal power as the proportion of simulation replicates (under the alternative hypothesis) with $P$ values $<2.5 \times 10^{-6}$. We estimated empirical power as the proportion of simulated datasets with $P$-values less than the estimated empirical threshold: the $\alpha$ th quantile of the $10^{8}$ $P$-values for the simulated samples under the null hypothesis. Overall, power using nominal or empirical thresholds yield near-identical power because tests are relatively wellcalibrated across all scenarios, and the empirical thresholds (range $\left.=1 \times 10^{-6}-3.5 \times 10^{-6}\right)$ are very similar to the nominal significance threshold. The greatest difference between nominal and empirical power is for a study with 100 males and 900 females in cases, and 500 males and 500 females in controls, where $50 \%$ of low-frequency variants are causal and have the same direction of effect. For the SKAT test using 


\section{X-Inactivation vs. Non-X-Inactivation Coding}

Burden Test

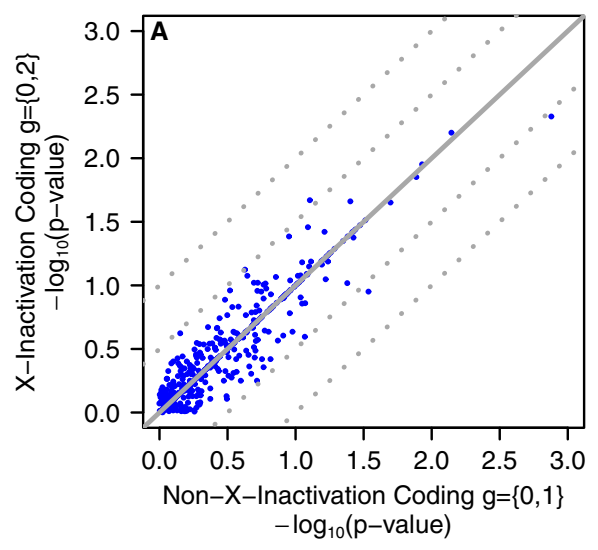

Burden

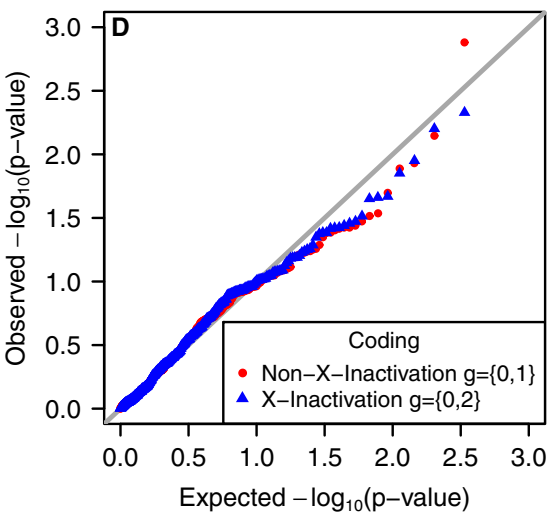

SKAT

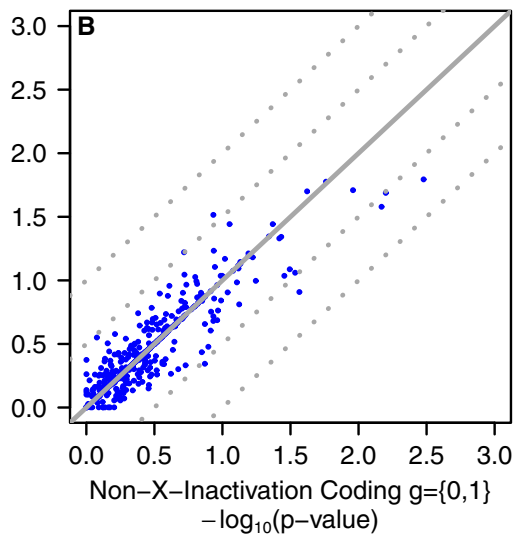

Quantile-Quantile Plots

SKAT

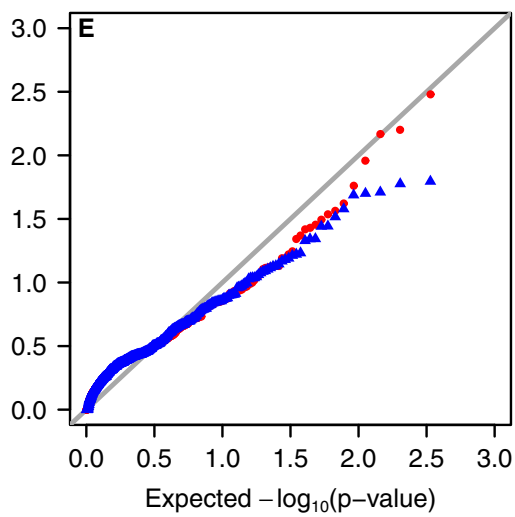

SKAT-O

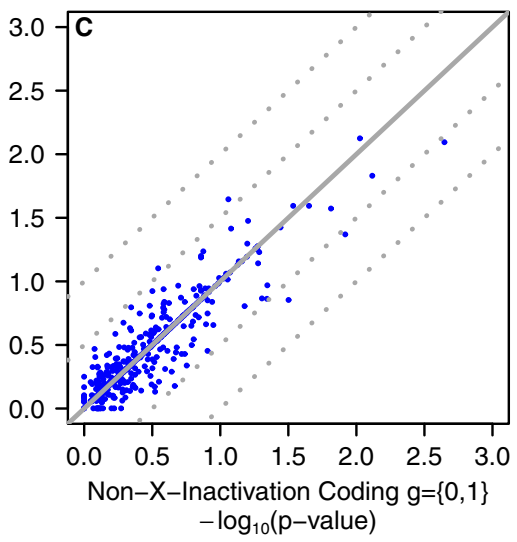

SKAT-O

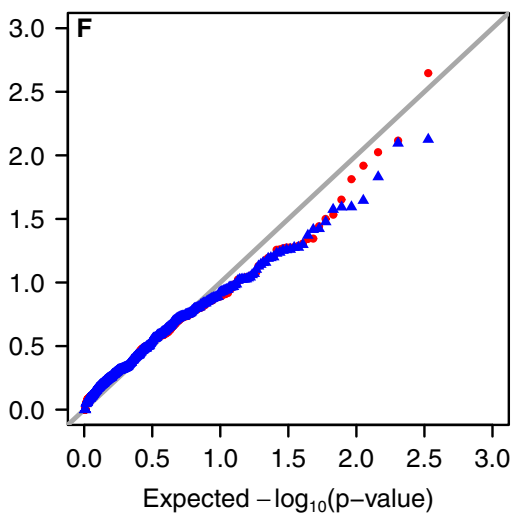

Difference in $\mathrm{X}$-Inactivation and Non-X-Inactivation $\mathbf{P}$-values

Burden

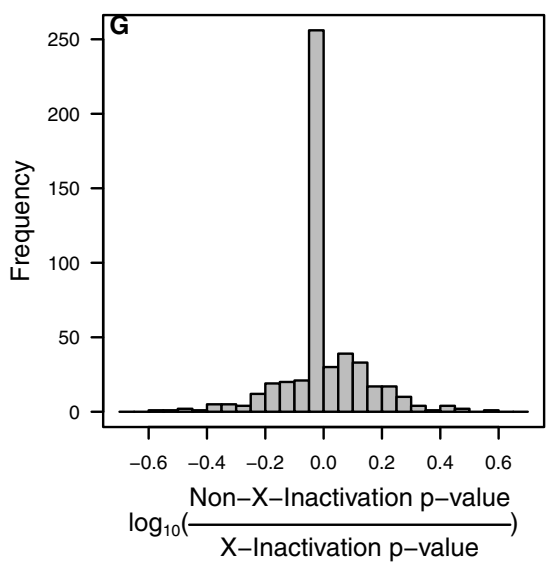

SKAT

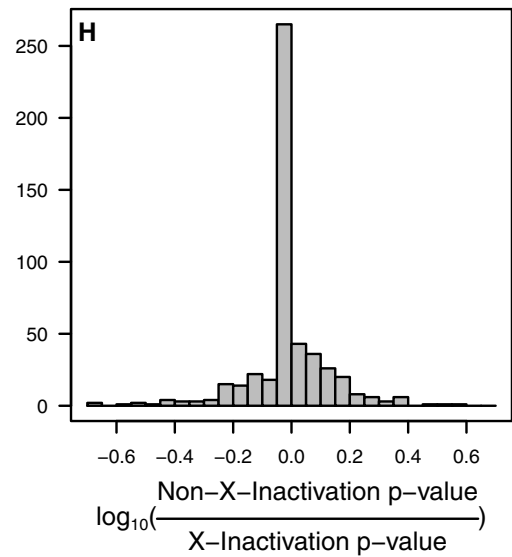

SKAT-O

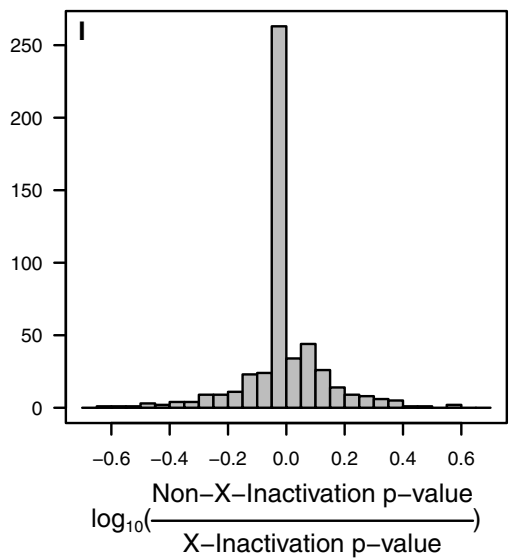

Figure 5. Comparison of association analysis $P$-values in the GoT2D study. (A-C) Scatterplots compare analysis $P$-values using non-X-inactivation $g=\{0,1\}$ and X-inactivation $g=\{0,2\}$ coding schemes for male genotypes. (D-F) Quantile-quantile plots compare expected and observed $P$-value distributions. (G-I) Histograms display the difference in $P$-values between the coding schemes. 
X-inactivation coding $\left(g_{i j}=\{0,2\}\right)$, nominal power $=41.7 \%$ vs. empirical power $=40.1 \%$. We decided to present our power results assuming nominal thresholds, because this is how analysis actually will be done.

We did not evaluate the calibration and power for other gene-based association methods, such as the WST [Madsen and Browning, 2009], C-alpha [Neale et al., 2011], and SSU [Pan, 2009] tests. However, our burden test is equivalent to the WST, and SKAT includes the C-alpha and SSU tests as a special case, indicating that our results would likely extend to these gene-based tests. We only evaluate test calibration and power assuming an additive genetic model. For gene-based tests, we are primarily interested in testing the (joint) effect of rare variants. Because minor allele homozygotes are rare, we expect the dominant genetic model to have near identical calibration and power to the additive model, and the recessive model to have very low power. We only explored the effect of random $\mathrm{X}$-inactivation, where $\sim 50 \%$ of the cells have one female allele inactivated and the remaining $\sim 50 \%$ of the other. We did not examine the possibility of nonrandom or skewed $\mathrm{X}$-inactivation [Amos-Landgraf et al., 2006], where $>75 \%$ of cells have one allele inactivated. Although a unified approach to account for both random and nonrandom $\mathrm{X}$-inactivation may be more robust and powerful, as demonstrated by Wang et al. [2014] for single-marker association testing, we speculate that misspecifying the model for the burden, SKAT, and SKAT-O gene-based tests will only result in a small power loss.

In conclusion, we generalized the burden, SKAT, and SKAT$\mathrm{O}$ tests to analyze $\mathrm{X}$-chromosome variants, and demonstrated that these tests are generally well-calibrated and powerful for a wide range of simulation scenarios. These tests can be directly applied to the association analysis of less common variants on the $\mathrm{X}$ chromosome.

\section{Acknowledgments}

We thank Laura J. Scott, Gonçalo Abecasis, and Cristen Willer for their helpful discussions and suggestions for the manuscript, and our GoT2D colleagues for allowing us to use sequencing data. This research was supported by the National Institutes of Health grants HG000376 and DK062370 to M.B. and HL113164 to S.L.

\section{References}

Amos-Landgraf JM, Cottle A, Plenge RM, Friez M, Schwartz CE, Longshore J, Willard HF. 2006. X chromosome-inactivation patterns of 1,005 phenotypically unaffected females. Am J Hum Genet 79:493-499.

Asimit J, Zeggini E. 2010. Rare variant association analysis methods for complex traits. Annu Rev Genet 44:293-308.
Carrel L, Willard HF. 2005. X-inactivation profile reveals extensive variability in Xlinked gene expression in females. Nature 434:400-404.

Clayton D. 2008. Testing for association on the X chromosome. Biostatistics 9:593-600.

Hickey PF, Bahlo M. 2011. X chromosome association testing in genome wide association studies. Genet Epidemiol 35:664-670.

Hindorff LA, MacArthur J, Wise A, Junkins HA, Hall PN, Klemm AK, Manolio TA. 2012. A catalog of published genome-wide association studies. NHGRI. Available at: www.genome.gov/gwastudies

Howie B, Fuchsberger C, Stephens M, Marchini J, Abecasis GR. 2012. Fast and accurate genotype imputation in genome-wide association studies through pre-phasing. Nat Genet 44:955-959.

Huyghe JR, Jackson AU, Fogarty MP, Buchkovich ML, Stancakova A, Stringham HM, Sim X, Yang L, Fuchsberger C, Cederberg H, and others. 2013. Exome array analysis identifies new loci and low-frequency variants influencing insulin processing and secretion. Nat Genet 45:197-201.

Lee S. 2014. SKAT: SNP-set (Sequence) Kernel Association Test. R package version 1.0.5. Available at: http://CRAN.R-project.org/package=SKAT.

Lee S, Emond MJ, Bamshad MJ, Barnes KC, Rieder MJ, Nickerson DA, Team NGESPELP, Christiani DC, Wurfel MM, Lin X. 2012. Optimal unified approach for rarevariant association testing with application to small-sample case-control wholeexome sequencing studies. Am J Hum Genet 91:224-237.

Lee S, Abecasis GR, Boehnke M, Lin X. 2014. Rare-variant association analysis: study designs and statistical tests. Am J Hum Genet 95:5-23.

Loley C, Ziegler A, Konig IR. 2011. Association tests for X-chromosomal markers-a comparison of different test statistics. Hum Hered 71:23-36.

Lyon MF. 1961. Gene action in the X-chromosome of the mouse (Mus musculus L.). Nature 190:372-373.

Madsen BE, Browning SR. 2009. A groupwise association test for rare mutations using a weighted sum statistic. PLoS Genet 5:e1000384.

Marchini J, Howie B, Myers S, McVean G, Donnelly P. 2007. A new multipoint method for genome-wide association studies by imputation of genotypes. Nat Genet 39:906-913.

Neale BM, Rivas MA, Voight BF, Altshuler D, Devlin B, Orho-Melander M, Kathiresan S, Purcell SM, Roeder K, Daly MJ. 2011. Testing for an unusual distribution of rare variants. PLoS Genet 7:e1001322.

Pan W. 2009. Asymptotic tests of association with multiple SNPs in linkage disequilibrium. Genet Epidemiol 33:497-507.

Price AL, Patterson NJ, Plenge RM, Weinblatt ME, Shadick NA, Reich D. 2006. Principal components analysis corrects for stratification in genome-wide association studies. Nat Genet 38:904-909.

Purcell S, Neale B, Todd-Brown K, Thomas L, Ferreira MA, Bender D, Maller J, Sklar P, de Bakker PI, Daly MJ, and others. 2007. PLINK: a tool set for whole-genome association and population-based linkage analyses. Am J Hum Genet 81:559-575.

Schaffner SF, Foo C, Gabriel S, Reich D, Daly MJ, Altshuler D. 2005. Calibrating a coalescent simulation of human genome sequence variation. Genome Res 15:15761583 .

Steinthorsdottir V, Thorleifsson G, Sulem P, Helgason H, Grarup N, Sigurdsson A, Helgadottir HT, Johannsdottir H, Magnusson OT, Gudjonsson SA, and others. 2014. Identification of low-frequency and rare sequence variants associated with elevated or reduced risk of type 2 diabetes. Nat Genet 46:294-298.

Wang J, Yu R, Shete S. 2014. X-chromosome genetic association test accounting for $\mathrm{X}$-inactivation, skewed $\mathrm{X}$-inactivation, and escape from X-inactivation. Genet Epidemiol 38:483-493.

Wise AL, Gyi L, Manolio TA. 2013. eXclusion: toward integrating the X chromosome in genome-wide association analyses. Am J Hum Genet 92:643-647.

Wu MC, Lee S, Cai T, Li Y, Boehnke M, Lin X. 2011. Rare-variant association testing for sequencing data with the sequence kernel association test. Am J Hum Genet 89:82-93.

Zhan X, Liu DJ. 2013. TaSer (TabAnno and SeqMiner): a toolset for annotating and querying next-generation sequence data. Available at: http://zhanxw. com/seqminer.

Zheng G, Joo J, Zhang C, Geller NL. 2007. Testing association for markers on the X chromosome. Genet Epidemiol 31:834-843. 